\title{
The Sondheimer-Wilson-Kohler Formula in Platinum Resistance Thermometry
}

\author{
R. J. Corruccini \\ Institute for Materials Research, National Bureau of Standards, Boulder, Colo.
}

(June 7, 1965)

\begin{abstract}
An attempt was made to determine the ideal resistivity versus temperature function for platinum by a parameter-variation method using the Sondheimer-Wilson-Kohler formula for the deviation from Matthiessen's rule. At the same time a test of the applicability of the SWK formula for practical thermometry was made by examining the accuracy with which it could fit the resistance-temperature functions of selected thermometers, the characteristics of which were restricted in a manner believed to favor their accurate representation. No ideal resistivity function could be found such that most of the thermometers could be accurately represented.
\end{abstract}

Key Words: Cryogenic, electrical resistivity, low temperature, platinum, resistance thermometry, temperature measurement, thermometry.

\section{Introduction}

The resistivity of a metal can be expressed by the formula

$$
\rho_{T}=\rho_{0}+\rho_{i T}+\Delta_{T}
$$

where $\rho_{0}$ is the imperfection-dependent resistivity at $T=0, \rho_{i T}$ is the temperature-dependent "ideal" resistivity of the hypothetical perfect lattice, and $\Delta_{T}$ is a small term resulting from the interaction of defect and lattice scattering of the electrons. Sondheimer and Wilson [1] ${ }^{1}$ showed on the basis of a two conductionband model that

$$
\Delta_{T}=\frac{\rho_{i T} \rho_{0}}{a \rho_{i T}+b \rho_{0}}
$$

and Kohler [2] obtained the same formula on more general grounds. In either treatment, the coefficients $a$ and $b$ are positive quantities and may be functions of temperature, but their forms have not been established from theory.

Equation (2), with $a$ and $b$ taken to be constants, was first applied to platinum resistance thermometry by Schultz [3]. It has recently received considerable attention as a device for establishing a platinum resistance scale of low temperatures [4]. A very extensive analysis of its application to this field has been made by Berry [5] who showed that it did not apply with sufficient accuracy to most of the thermometers considered or even to most of those of high purity; i.e., having high values of the coefficient, $\alpha \equiv\left(R_{373.15}\right.$ $\left.-R_{273.15}\right) / 100 R_{273.15}$, or low values of the reduced residual resistance, $W_{0} \equiv R_{0} / R_{273.15}$.

\footnotetext{
${ }^{1}$ Figures in brackets indicate the literature references at the end of this paper.
}

In this paper we attempt to determine the ideal resistivity function by a new method; also we test the applicability of the SWK formula to a rather homogeneous group of high-purity thermometers that have been previously correlated by other means [6]. Some preliminary work along the lines of the present paper was reported earlier [6].

\section{Procedures}

\subsection{Reduced Form of the SWK Equation}

Equations (1) and (2) with $a$ and $b$ taken to be constants are first transformed into relations involving resistance ratios. We follow Schultz [3] but use the notation of Berry [5] where applicable. Equation (1) becomes

$$
W_{T}=W_{i T}+W_{0}\left(1-W_{i T}\right)\left(1+\Gamma_{T}\right)
$$

where

$$
\begin{aligned}
W_{T} & \equiv R_{T} / R_{273.15} \\
W_{i T} & \equiv R_{i T} / R_{i 273.15} \\
W_{0} & \equiv R_{0} / R_{273.15}
\end{aligned}
$$

and

$$
\Gamma_{T}=\left(\Delta_{T}-\Delta_{273.15} W_{i T}\right) / \rho_{273.15} W_{0}\left(1-W_{i T}\right) .
$$

Introduction of eq (2) leads to

$$
\left(\Gamma_{T}\right)_{\mathrm{SWK}}=A W_{i T} /\left(1+C W_{i T}\right)
$$

in which $A$ and $C$ are constants which can be evaluated 
if the resistance is known at any two temperatures, $T_{1}$ and $T_{2}$, other than 0 and $273.15^{\circ} \mathrm{K}$. The following expressions are found for $A$ and $C$ :

$$
\begin{aligned}
& C=\frac{\Gamma_{1}-\Gamma_{2} W_{i 1} / W_{i 2}}{\left(\Gamma_{2}-\Gamma_{1}\right) W_{i 1}} \\
& A=\Gamma_{2}\left(1+C W_{i 2}\right) / W_{i 2} .
\end{aligned}
$$

The subscripts 1 and 2 refer, respectively, to $T_{1}$ and $T_{2}$. The constants $A$ and $C$ are related to the SWK parameters by the following close approximations:

$$
\begin{aligned}
& a \approx C / A \\
& b \approx 1 / A W_{0} .
\end{aligned}
$$

In the calculations to be described, $T_{1}$ and $T_{2}$ were always taken to be 20 and $90^{\circ} \mathrm{K}$; i.e., approximately the boiling points of hydrogen and oxygen, respectively.

\subsection{The Thermometers}

These consisted of 34 commercially made capsuletype thermometers plus thermometer T4 of the National Research Council, Canada. They are more fully described in the earlier paper [6]. All of the calibration data were expressed on the NBS 1955 temperature scale. The group is of high purity as indicated by $\alpha>0.003924$ and $W_{0}$ (estd.) $<7 \times 10^{-4}$. The success with which they were correlated by the simpler empirical method of reference 6 suggested that they were rather uniform with regard to the forms of their deviations from Matthiessen's rule and thus would provide as favorable a practical test of the SWK formula as one could reasonably hope to obtain. The thermometers are referred to by identification numbers from 1 to 35 assigned in order of increasing $W_{20.263}$.

\subsection{The Residual Resistance Ratio $W_{\circ}$}

Experimental values of $W_{4.2}$ were available for five thermometers. These were adjusted to $W_{0}$ using the data in Berry's figure 1. For the remaining ones, $W_{0}$ was estimated from data in the region 10 to $20^{\circ} \mathrm{K}$ using Method 1 in Berry's appendix III.

\subsection{The Ideal Resistance Ratio Function $W_{i T}$}

The function $W_{i T}$ for platinum has been estimated by various methods. A summary and discussion was given by Berry who indicated objections to the various earlier methods. He derived a new function, slightly different from the others. In the region 10 to $90{ }^{\circ} \mathrm{K}$, this was found by extrapolating plots of $W_{T}-W_{0}$ versus $W_{0}$ for a large number of thermometers to $W_{0}=0$. Since the values of $W_{T}-W_{0}$ unfortunately lay in a band rather than on a line, only a few selected thermometers having low values of $W_{T}-W_{0}$ were used in making the extrapolation. Correspondingly, when
Berry fitted the SWK formula using this $W_{i T}$ function to the various thermometers, the fit was satisfactory only for those few that had been used to derive the $W_{i T}$ function.

The validity of the SWK model for the present group of thermometers is here tested by a different method, as follows. One may derive various arbitrary $W_{i T}$ functions from the $W_{T}$ function of any one thermometer via eqs (3) and (4) by assuming various values of its parameters $A$ and $C$. Equations (3) and (4) can then be fitted to all of the remaining thermometers at 20 and $90{ }^{\circ} \mathrm{K}$ using these various $W_{i T}$ functions in turn, and the errors in the representations of $W_{T}$ at other temperatures can be examined. If it is supposed that the SWK formula holds for the group, then it should be possible to find in this way a particular $W_{i T}$ function such that all of the remaining thermometers except the one used to generate the $W_{i T}$ functions are accurately fitted. Furthermore if various thermometers are used in turn to generate such an optimum $W_{i T}$ function, then the various optimum $W_{i T}$ functions should be found to be all identical and therefore equal to the "true" $W_{i T}$ function.

To carry out this test, nine thermometers were selected which, on the basis of earlier work [6], were judged to be representative of the set of thirty-five. The five of these for which experimental values of $W_{4.2}$ were available were used to generate the trial $W_{i T}$ functions.

\section{Results}

\subsection{The Function $W_{i T}$}

When the procedure just described was carried out it was found that there existed a spectrum of suitable $W_{i T}$ functions. In going from one to another of these functions, the identities of the thermometers for which the fit was least satisfactory changed, as did the temperatures at which the errors were largest, but no uniquely superior fit of all the thermometers could be found. In figure 1 are shown some of the better $W_{i T}$ functions referenced to Berry's $W_{i T}$ function as a base line.

The fitting errors (see the next section) were roughly an order of magnitude larger than the precision with which the resistance thermometers are believed to have been calibrated. Consequently, the variation in $W_{i T}$ found by the present method is an artifact of the SWK model and is not chargeable to experimental error.

\subsection{Accuracy of the SWK Equation With Constant Coefficients}

The quality of the better fits obtained using $W_{i T}$ functions derived by the method of section 2.4 may be summarized by stating that the maximum temperature error of the nine representative thermometers in the interval, 20 to $273.15^{\circ} \mathrm{K}$, was never much less than 20 mdeg, and the average of the maximum errors of the thermometers was never much less than $10 \mathrm{mdeg}$. In 


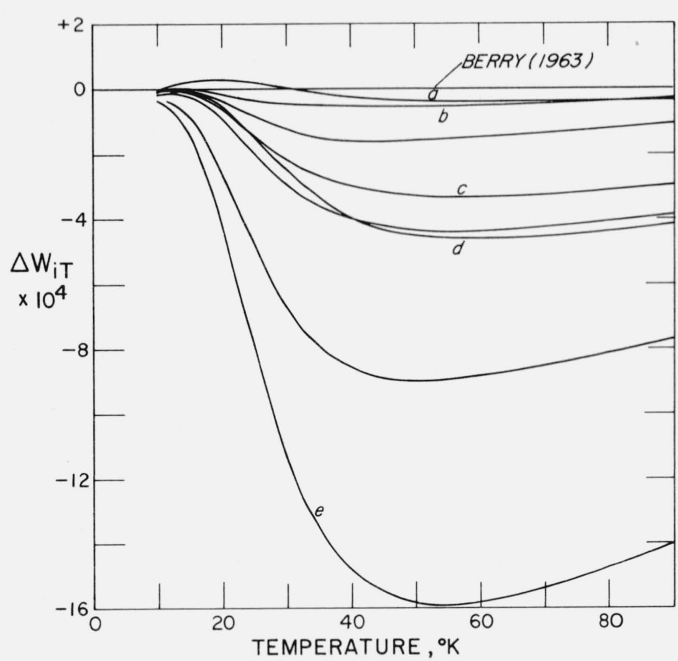

FIGURE 1. Selected useable $\mathrm{W}_{\mathrm{iT}}$ functions plotted relative to that of Berry [5].

(The letters, a-b-c-d-e, identify functions referred to in table 1.)

table 1 are summarized the errors of the SWK formula resulting from use of five of the better fitting $W_{i T}$ functions of figure 1. For comparison, Berry's $W_{i T}$ function was also used. In the last two rows are given the corresponding errors of the simple empirical method evaluated earlier [6].

TABLE 1. Accuracy of the SWK formula compared to an empirical method of interpolation

\begin{tabular}{|c|c|c|c|}
\hline Method of interpolation & Max. temp. error & Average $\left|\Delta T_{\max }\right|^{\text {a }}$ & Range examined \\
\hline $\begin{array}{l}\text { SWK eqs }(3-4) \text { using } \\
\text { the following } W_{i T} \\
\text { functions: } \\
\text { a, Figure 1 } \\
\text { b. Figure 1 } \\
\text { c, Figure 1 } \\
\text { d, Figure 1 } \\
\text { e. Figure 1 } \\
\text { Berry [5] } \\
\text { Berry [5] }\end{array}$ & $\begin{array}{l}\text { mdeg } \\
-17.0 \\
+20.3 \\
+21.3 \\
-18.4 \\
-21.7 \\
+27.1 \\
+27.1\end{array}$ & $\begin{array}{r}\text { mdeg } \\
8.5 \\
12.4 \\
10.6 \\
8.6 \\
10.6 \\
12.8 \\
12.7\end{array}$ & \begin{tabular}{c}
\multicolumn{1}{c}{${ }^{\circ}$} \\
$20-273$ \\
$20-273$ \\
$20-273$ \\
$20-273$ \\
$20-273$ \\
$20-273$ \\
$20-90$
\end{tabular} \\
\hline $\begin{array}{l}\text { Empirical method }{ }^{\mathrm{b}} \\
\text { Do............................... }\end{array}$ & $\begin{array}{l}+8.6 \\
-4.8\end{array}$ & $\begin{array}{l}3.6 \\
2.2\end{array}$ & $\begin{array}{l}20-273 \\
20-90\end{array}$ \\
\hline
\end{tabular}

a Average of the maximum errors of the nine representative thermometers

${ }^{\mathrm{b}}$ Reference 6, eq (10) using 20.90 , and $273.15^{\circ} \mathrm{K}$ as calibration points.

All earlier studies of the resistance-temperature relation of platinum have encountered exceptional difficulties in representing it accurately in terms of temperature errors below $20^{\circ} \mathrm{K}$. It might reasonably be supposed that the SWK formula would be advantageous here because of its theoretical foundation and the constraint imposed on its behavior at the lowest temperatures by use of the residual resistance. However, its behavior below $20^{\circ}$ also is unsatisfactory inasmuch as the error at $10^{\circ} \mathrm{K}$ was usually of the order of $0.1 \mathrm{deg}$.

\subsection{Further Characterization of the Thermometers}

Following Berry, we may characterize the thermometers via the function $\left(\Gamma_{T}\right)_{\text {exptl }}$, calculated from eq (3) using the experimental values of $W_{T}$, i.e.,

$$
\left(\Gamma_{T}\right)_{\operatorname{exptl}} \equiv \frac{W_{T}-W_{i T}}{\left(1-W_{i T}\right) W_{0}}-1
$$

This function, if it is to be fitted by eq (4), must rise monotonically-at first in proportion to $W_{i T}$, i.e, roughly as $T^{5}$-and approach an asymptotic limit. Berry found that the thermometers which would be well represented could be identified as those with (a) $\Gamma_{90}<0.35 \quad\left(\Gamma_{90}\right.$ approximates the high-temperature limit of $\Gamma_{T}$ ), (b) $W_{0}<1 \times 10^{-3}$, (c) monotonically rising curves of $\Gamma_{T}$. Only about 20 percent of the thermometers examined by him met all three requirements.

It will be recognized that conditions (a) and (c) test only the uniformity of the group. They are not meaningful criteria of "normal" versus "anomalous" behavior inasmuch as the behavior displayed depends entirely on the form of the adopted $W_{i T}$ function.

The present group of thermometers was examined in the above way using Berry's $W_{i T}$ function. All of the thermometers met criterion (b). Eighteen out of the 35 met criterion (a). Fifteen of these 18, as well as two of the others, failed to strictly meet criterion (c). However only five of these (consisting of the thermometers with the lowest values of $\Gamma_{90}$ ) showed marked maxima in $\left(\Gamma_{T}\right)_{\text {exptl }}$. If a requirement based on calibration at common fixed points were to be adopted for the purpose of excluding thermometers having maxima in $\Gamma_{T}$ (e.g., $\Gamma_{90}>\Gamma_{54}>\Gamma_{27}>\Gamma_{20}$ ), only these five would be excluded. Had members of this set of five been excluded from the nine used for most of the calculations, the SWK fits would have been improved for certain of the $W_{i T}$ functions derived and selected by the parameter-variation method, but would still have been inferior to that given by the empirical method of reference 6 . The average fit of the SWK formula using Berry's $W_{i T}$ function would actually have worsened.

Figure 2 shows a sampling of the $\left(\Gamma_{T}\right)_{\text {exptl }}$ curves. Numbers $17,31,5,34,23$, and 11 are from the selected group of nine used for most of the calculations. Number 29 also was a typical curve; however, number 12 was unique. Numbers 11 and 29 provided reference functions used in the empirical method of reference 6 . Numbers 23,31 , and 34 were used in a preliminary study [7] in which it was found that they could be represented within about 1 mdeg by the method of reference 6 .

Table 2 lists the SWK parameters of the selected group of nine representative thermometers used in the derivation of $W_{i T}$ and in examining the fit of the SWK equations. It may be compared with Berry's table IV. The range of the parameters $a$ and $b$ is very nearly the same as that covered by Berry although the range of $W_{0}$ is much narrower. 


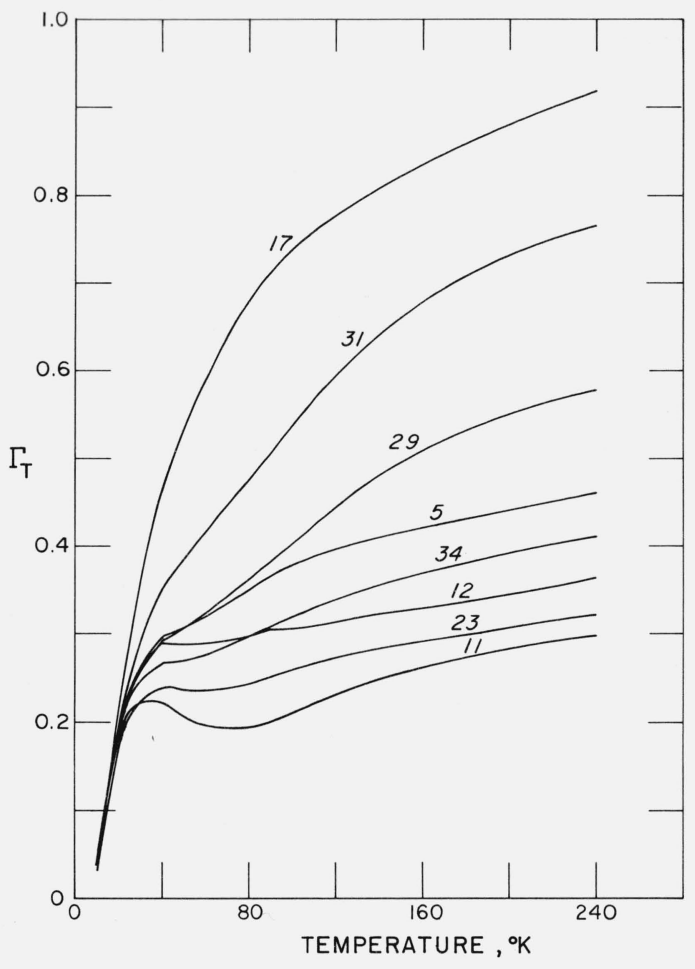

FigURE 2. Curves of $\left(\Gamma_{T}\right)_{\text {expt. }}$ for selected thermometers calculated using Berry's $\mathrm{W}_{\mathrm{iT}}$ function.

(The curves are labeled with the identification numbers of the thermometers.)

TABLE 2. SWK parameters of the selected thermometers

\begin{tabular}{c|c|c|c|c|c}
\hline \hline $\begin{array}{c}\text { Thermometer } \\
\text { number }\end{array}$ & $\mathrm{W}_{\mathbf{0}} \times 10^{\mathrm{6}}$ & $A$ & $C$ & $\frac{1}{a}\left(\approx \frac{A}{C}\right)$ & $\frac{b}{a}\left(\approx \frac{1}{C W_{0}}\right)$ \\
\hline & & 110 & 146 & 0.75 & 16.0 \\
$2^{\mathrm{a}}$ & 427 & 812 & 113 & .74 & 17.4 \\
17 & 646 & 86 & 166 & .52 & 9.0 \\
31 & 468 & 95 & 251 & .38 & 8.5 \\
34 & 681 & 135 & 430 & .32 & 3.4 \\
23 & 6555 & 134 & 529 & .25 & 2.9 \\
10 & 395 & 231 & 926 & .25 & 2.2 \\
1 & 398 & 386 & 662 & .22 & 3.8 \\
11 & 498 & 386 & 1980 & .19 & 1.0 \\
\hline
\end{tabular}

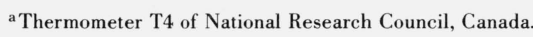

\section{Discussion and Conclusions}

The application of the SWK formula to platinum resistance thermometry has been based on the reasonable expectations that, because of its theoretical foundations, it should accurately represent a wider range of platinum purities and a wider range of temperatures than could purely empirical correlations. The work of Berry and others has already dashed these expectations. The failure of the present study to obtain a unique ideal resistivity function from the SWK model with constant coefficients as well as the failure to fit the model to any major part of the data used within the precision of those data indicate that the model has only qualitative significance. This supports the conclusions already reached.

Thus the present study is of use mainly in examining the question: Would the SWK formula have some special usefulness if the characteristics of the thermometers were narrowly restricted? The present thermometers have narrowly restricted $\alpha$ and $W_{0}$ values and are of uniform origin and style of construction. It has been shown that they can be accurately represented from 20 to $90{ }^{\circ} \mathrm{K}$ by a simple empirical method [6] using the same calibration temperatures as the present study except that the residual resistance is not required. Thus by these various criteria the present thermometers appear to be a quite homogeneous group. A correlation scheme that required any further restrictions on acceptable thermometer characteristics would not be justifiable on practical grounds. Yet the present study shows that the thermometers considered are a diverse group when categorized according to any of the following: (1) shape of the $\Gamma_{T}$ function obtained using Berry's $W_{i T}$ function, (2) magnitude of the SWK parameters, such as $a$, and (3) accuracy with which the SWK formula fits their resistance-temperature functions. Consequently, it seems unlikely that the SWK formula has any practical utility for accurate thermometry or for classification of thermometers into acceptable and unacceptable types.

The assistance of William J. Hall and Hans M. Roder in programming the calculations for automatic computation is gratefully acknowledged.

\section{References}

[1] E. H. Sondheimer and A. H. Wilson, Proc. Roy. Soc. (London), A190, 449 (1947).

[2] M. Kohler, Z. Physik 126, 495 (1949).

[3] H. Schultz, Z. angew. Physik 9, 465 (1957).

[4] See papers by Corruccini, Harper et al., Sharevskaya et al., and Van Dijk in Temperature, Its Measurement and Control in Science and Industry, C. M. Herzfeld, ed. (Reinhold Publishing Corp., New York, 1962), Vol. 3, Part 1, Section VII, Platinum Resistance Thermometer Scale, 10 to $90{ }^{\circ} \mathrm{K}$.

[5] R. J. Berry, Can. J. Phys. 41, 946 (1963).

[6] R. J. Corruccini, Temperature, Its Measurement and Control in Science and Industry, C. M. Herzfeld, ed. (Reinhold Publishing Corp., New York, 1962), Vol. 3, Part 1, p. 329.

[7] R. J. Corruccini, Rev. Sci. Instr. 31, 637 (1960). 